\title{
Significance of Virchow-Robin Spaces in Patients Newly Diagnosed with Multiple Sclerosis: A Case-Control Study in an Arab Population
}

\author{
Osama Al-Saeed ${ }^{a} \quad$ Reji Athyal $^{b}$ Mohammed Ismail ${ }^{c}$ Mehraj Sheikh ${ }^{a}$ \\ a Department of Radiology, Faculty of Medicine, Health Sciences Centre, Kuwait University, Jabriya, \\ ${ }^{b}$ Department of Radiology, Al-Amiri Hospital, Sharq, and ' Department of Radiology, Ibn Sina Hospital, \\ Shuwaikh, Kuwait
}

\section{Key Words}

Magnetic resonance imaging • Multiple sclerosis •

Virchow-Robin spaces $\cdot$ Arabs

\begin{abstract}
Objective: To assess the frequency and extent of dilatation of Virchow-Robin (VR) spaces at three levels of the brain in patients of Arab ethnicity in Kuwait recently diagnosed with multiple sclerosis (MS) and compare the results with ageand gender-matched controls. Methods: The magnetic resonance imaging (MRI) scans performed within 3 months of the clinical diagnosis of 80 patients recently diagnosed with active MS were compared to those of 80 age- and gendermatched controls with headache but without any neurological deficits for the frequency and size of VR spaces. MRI was done with noncontrast axial and coronal $\mathrm{T}_{1} \mathrm{~W} F \mathrm{FS}$, axial $\mathrm{T}_{2} \mathrm{~W}$ FSE, axial $T_{2} W$ FLAIR and sagittal FLAIR sequences followed by postcontrast axial and coronal $\mathrm{T}_{1} \mathrm{~W}$ sequences. The frequency of VR spaces in MS patients and controls at midbrain, lenticulostriate vessels and supraventricular levels was analyzed using a two-tailed McNemar test. Results: There was no difference in the frequency of VR spaces at the levels of the midbrain, lenticulostriate vessels and supraventricular white matter between MS patients and controls. In the supraventricular region, however, there were 91 dilated VR
\end{abstract}

spaces in 26 (32\%) of the MS patients while in the control group, there were 8 dilated VR spaces in $6(7.5 \%)$ patients and the difference was statistically significant $(p<0.0001)$. Conclusion: The data showed that dilated VR spaces in the supraventricular region could potentially be used as a marker for MS and as a prognostic tool. However, further studies with a larger population are needed to further evaluate and confirm this observation.

Copyright $\odot 2012$ S. Karger AG, Basel

\section{Introduction}

Virchow-Robin (VR) spaces are pia-lined extensions of the subarachnoid space surrounding the small arteries and arterioles that perforate the surface of the brain and extend into the substance of the brain parenchyma [1]. Vascular ectasia and tortuosity, increased vessel wall permeability, decreased vascular compliance, ex-vacuo change in cortical atrophy and ischemic perivascular tissue loss are believed to be responsible for dilatation of VR spaces $[2,3]$. Several researchers have described the value of VR spaces in a number of disease processes such as small vessel disease, dementia and hypertension [4-6], but few have analyzed the relationship between VR spaces and multiple sclerosis (MS) [7-9]. 
Table 1. Frequency of nondilated VR spaces (VRS) in the brain parenchyma in MS patients and controls

\begin{tabular}{|c|c|c|c|c|c|c|}
\hline & \multicolumn{2}{|l|}{ Level 1} & \multicolumn{2}{|l|}{ Level 2} & \multicolumn{2}{|l|}{ Level 3} \\
\hline & MS & control & MS & control & MS & control \\
\hline Grade 0 (0 VRS) & $72(90 \%)$ & $75(93.8 \%)$ & $6(7.5 \%)$ & $4(5.0 \%)$ & $32(40 \%)$ & $12(15 \%)$ \\
\hline Grade 1 (1-3 VRS) & $8(10 \%)$ & $5(6.2 \%)$ & $41(51.25 \%)$ & $53(66.3 \%)$ & $8(10 \%)$ & $19(23.9 \%)$ \\
\hline Grade 2 (4-7 VRS) & 0 & 0 & $31(38.75 \%)$ & $23(28.89 \%)$ & $20(25 \%)$ & $25(31.3 \%)$ \\
\hline Grade 3 (>7 VRS) & 0 & 0 & $2(2.5 \%)$ & 0 & $20(25 \%)$ & $24(30.3 \%)$ \\
\hline
\end{tabular}

Level 1 = Mid-brain; level 2 = lenticulostriate nucleus; level 3 = supraventricular region.

MS is a chronic debilitating disease of the central nervous system believed to be immune-mediated, and although its etiology is still unknown, possibly related to a combination of genetic and environmental factors [7]. Most MS lesions have been shown to be in a perivenous location or on pial and ependymal surfaces. An accumulation of inflammatory cells in VR spaces is an important characteristic in acute lesions [10]. It has been suggested in previous studies that dilated perivascular spaces could be more predominant in patients with this disease [7-9]. Hence, the aim of our study was to determine the frequency and degree of dilatation of VR spaces at three levels in patients of Arab ethnicity newly diagnosed with MS and evaluate whether there is a statistically significant difference in the number and size of VR spaces in this group.

\section{Subjects and Methods}

This was a case-control study of 80 patients (age range 1549 years) who fulfilled the criteria of recent onset of MS, all of whom had undergone magnetic resonance imaging (MRI) within 3 months of initial presentation (between January 2010 and February 2011) with neurological symptoms clinically suggestive of MS. Patients were diagnosed using McDonald criteria for MS following clinical evaluation. MRI studies and other clinical tests including cerebrospinal fluid (CSF) analysis were done when required. Eighty individuals were age- and gender-matched to controls who presented with headache without any neurological deficits and who also had normal MRI studies performed within the same period (fig. 1).

Brain MRI was done on a 1.5-tesla machine (GE Signa 1.5T Echospeed) using an 8-channel head coil. The noncontrast sequences included the axial and coronal $\mathrm{T}_{1} \mathrm{~W} F S E$, axial $\mathrm{T}_{2} \mathrm{~W}$ FSE, axial $\mathrm{T}_{2} \mathrm{~W}$ FLAIR and sagittal FLAIR sequences. After intravenous contrast by manual injection of $0.1 \mathrm{mmol} / \mathrm{kg}$ body weight of gadodiamide (Omniscan, GE Health Care, Cork, Ireland), axial and coronal $\mathrm{T}_{1} \mathrm{~W}$ FSE sequences were performed.
The VR spaces were identified using the following criteria: punctate foci that were of CSF intensity on all pulse sequences; areas conforming to the pathway of the penetrating arteries; areas that had no mass effect, as previously described [11, 12]. The absence of any rim of hyperintensity on the FLAIR images was used to differentiate VR spaces from old lacunar infarctions [4].

The frequency of VR spaces was evaluated at three levels in the brain parenchyma: level 1 - the midbrain; level 2 - the lenticulostriate nucleus, and level 3 - the supraventricular region (fig. 1). A grading scale was applied ranging from $0=$ no VR spaces to $1=1-3,2=4-7$, and $3=>7$ VR spaces as described by Achiron and Faibel [9]. The side with more VR spaces at each level was used for grading [13]. VR spaces were considered to be dilated if they were $\geq 2 \mathrm{~mm}$ [9]. As above, the side with more dilated VR spaces was used in the analysis. Statistical analysis was performed using the two-tailed McNemar test.

\section{Results}

At the three evaluated levels, a greater number of VR spaces were noted in the supraventricular region with 20 (25\%) of 80 patients with MS and $24(30.3 \%)$ of 80 subjects who had a frequency of grade $3(>7)$. At the midbrain level a large number of patients (72, 90\%) and controls (75-93.8\%) did not have any VR spaces. The greatest number of VR spaces at the level of the lenticulostriate vessels was noted to be grade 1 (1-3 VR spaces) in both patients and controls. Importantly, there was no increase noted in the frequency of nondilated VR spaces in the MS group compared to the control group at all levels. In fact, the number was smaller in MS patients compared to controls in the supraventricular region. At the other two levels, the number was comparable (table 1).

There was no difference between MS patients and controls at levels 1 and 2 in the number of dilated VR spaces with no dilated VR spaces in patients or controls 

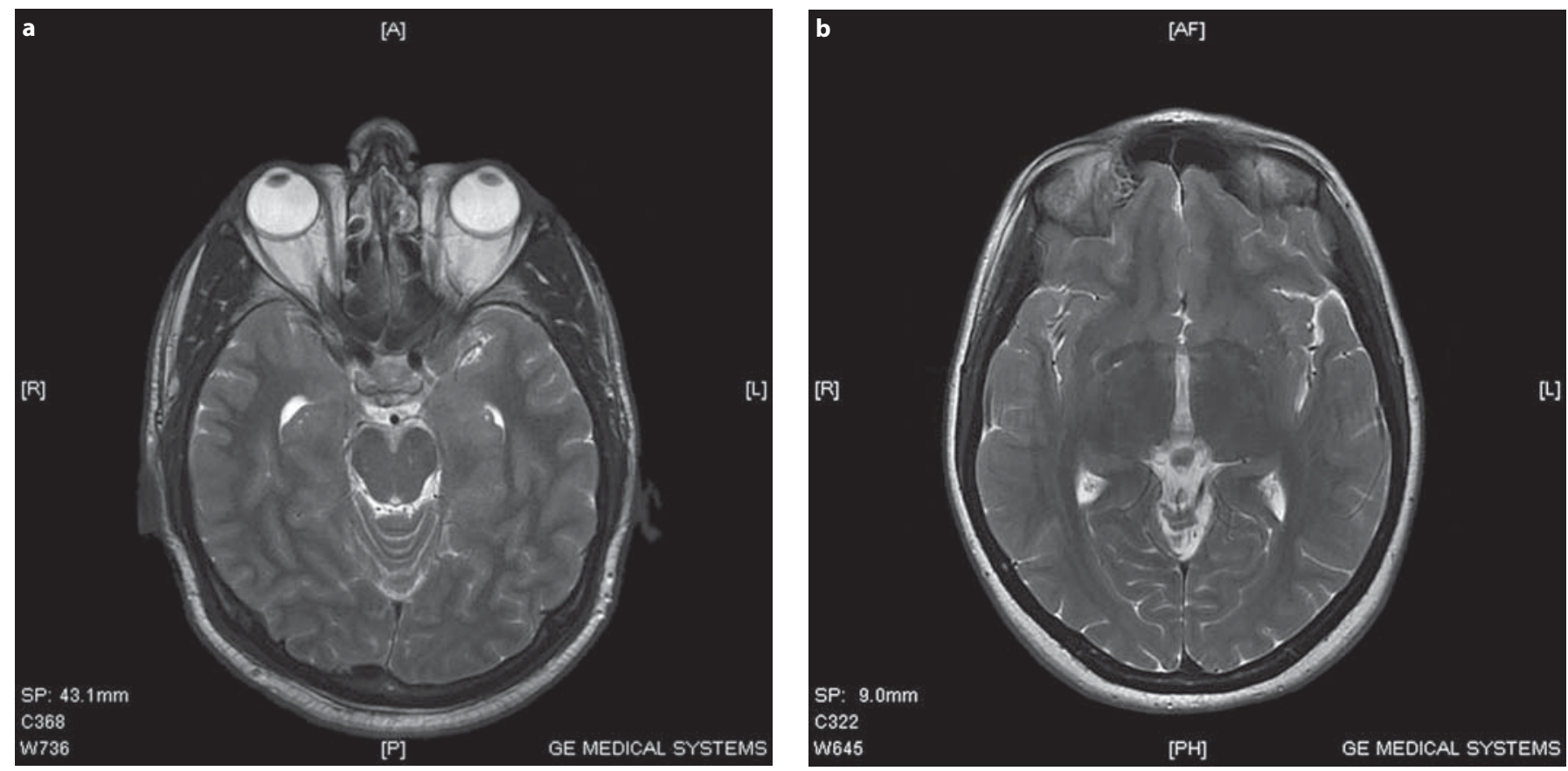

Fig. 1. Axial $\mathrm{T}_{2} \mathrm{WI}$ at midbrain level (a), lentiform nucleus (b) and supraventricular level (c) showing tiny punctate normal VR spaces.

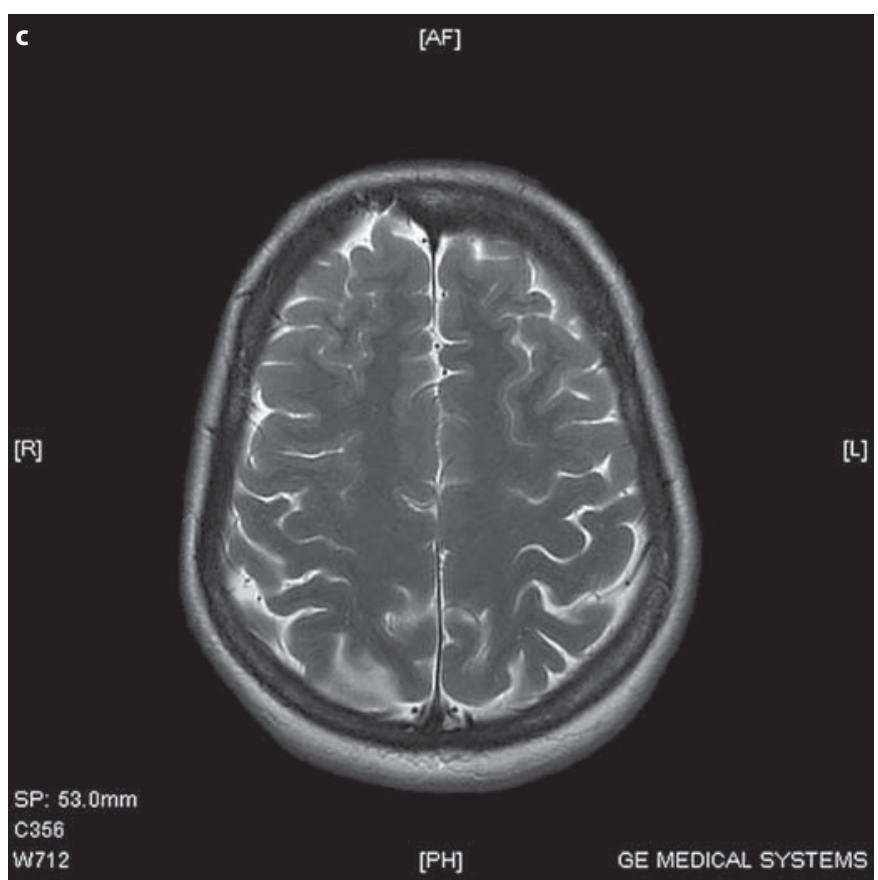

at the level of the midbrain and a total of 24 and 25 dilated VR spaces, respectively, in patients and controls at the level of the lenticulostriate nucleus. At level 3 (supraventricular), there were 91 dilated VR spaces in 26 (32\%) patients of the MS group, between 2 and 6 in each patient

(fig. 2). No specific age or gender distribution was noted. In the control group, there were 8 (7.5\%) dilated VR spaces in 6 patients, between 1 and 2 in each. The calculated $\mathrm{p}$ value for dilated VR spaces at the supraventricular level was $<0.0001$. 


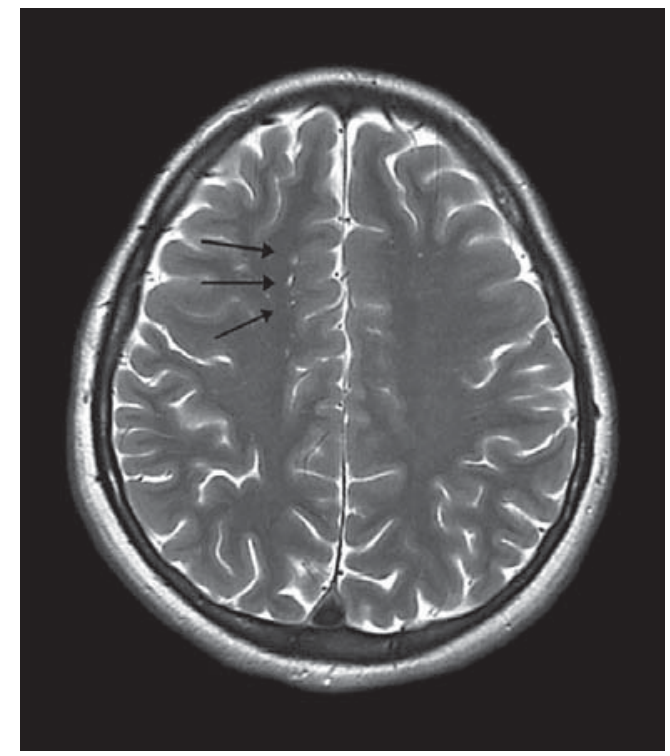

Fig. 2. Axial $\mathrm{T}_{2} \mathrm{WI}$ at the supraventricular level in a patient with MS showing a group of dilated VR spaces (arrows).

\section{Discussion}

In our study, in the supraventricular region, there was a significantly larger number of dilated VR spaces noted in patients with recently diagnosed MS than in the controls.

There was, however, no significant increase in the frequency of VR spaces in MS patients compared to controls at the midbrain level, basal ganglia and in the supraventricular region.

VR spaces are potentially one of the most important entry routes of soluble proteins as well as of leukocytes into the central nervous system [14], believed to be associated with the transfer of soluble factors between the extracellular fluid of the brain and the CSF. This has been elucidated through ultrastructural studies and also through the intracerebral injection of tracer substances [15]. Macrophages containing major histocompatibility complex antigens can trap foreign antigens entering the brain $[16,17]$. The accumulation of activated macrophages in large numbers and $\mathrm{T}$ and $\mathrm{B}$ lymphocytes can result in a significant immune response, as occurs in MS. The demyelinating lesions of MS are typically situated adjacent to veins or on pial and ependymal surfaces. There are cuffs of inflammatory cells present in VR spaces as a prominent feature of acute MS plaques, and this perivascular accumulation of cells could either be due to the resultant overflow of immune response or the stimu- lating antigens being confined to cells in the perivascular spaces [10].

There have been several studies that have found a higher incidence of dilated VR spaces in hypertension [5], white matter lesions of leukoaraiosis [18], dementia [6] and retinopathy in diabetics [19] and have suggested an association of dilated VR spaces with disease entities related to small vessel disease. In a recent study, Zhu et al. [20] evaluated a large cohort of elderly subjects and found a strong association between the severity of dilated VR spaces and age and hypertension. The severity was also related to the volume of white matter leukoaraiosis and lacunar infarctions as seen on MRI. They concluded that there appeared to be an independent association between the degree of dilatation of VR spaces and age, hypertension, volume of white matter hyperintensities, and lacunar infarctions, and accordingly suggested that dilated VR spaces be considered another marker of cerebral small vessel disease in the elderly. Animal studies have also revealed that in acute and chronic autoimmune encephalomyelitis (the animal model for MS) initially there was an increase in the number of inflammatory cells in the subarachnoid space, noticed even prior to any neurological impairment with accumulation of cells in the VR spaces observed subsequently $[17,21]$.

Wuerfel et al. [8] found a significantly larger size of VR spaces in patients with MS than in controls, which was also observed in our study. In addition, they demonstrated an increase in the size and number when contrast-enhancing lesions indicative of blood-brain barrier disruption appeared. However, in our study of a population of Arab ethnicity, we did not find an increased number of VR spaces at three levels as previously reported by Archiron et al. [9] and Etemadifar et al. [7]. The larger size of VR spaces noted in our study could be related to the infiltration of cells that occurs in patients with MS. During the generation and maintenance of inflammatory processes, as occurs in these patients, enlargement due to cell and fluid accumulation occurs that is easily demonstrated on MR images [22-25]. In a postmortem study in MS patients, Vos et al. [26] demonstrated enlarged VR spaces containing infiltrated leukocytes associated with diffusely abnormal white matter and focal abnormalities on postmortem MRI. Our observation of larger-size VR spaces in the supraventricular region rather than at the level of the basal ganglia or the midbrain could indicate a more significant role for perivascular macrophages and stimulating antigens in VR spaces and possibly a more active immune-mediated response in the convexity region. 
In our study, the finding of an increased size of VR spaces in the supraventricular region leads us to postulate that these spaces represent sites that serve as triggers for inflammatory activity in the central nervous system. The convexity region could potentially have a more significant role in the generation of inflammatory activity with infiltration of cells and edema compared to the other two levels. Larger studies with a greater number of cases and controls are needed to confirm our findings. It is likely that following up VR space size in specific locations and possibly assessing the change in size during phases of neurologically and radiologically active and inactive disease might demonstrate this observation more conclu- sively. These parameters could also be studied in relation to varying treatment protocols for MS. These are potential areas of further research.

\section{Conclusion}

The frequency of VR spaces was similar for MS and control groups of Arab ethnicity; however, this study indicates the presence of a significantly larger number of dilated VR spaces in the supraventricular region in patients with recently diagnosed MS. This might serve as a marker for the disease and could also serve as a prognostic tool.

\section{References}

1 Hutchings M, Weller RO: Anatomical relationships of the pia mater to cerebral blood vessels in man. J Neurosurg 1986;65:316325.

2 Barkof F: Enlarged Virchow-Robin spaces: do they matter? J Neurol Neurosurg Psychiatry 2004;75:1516-1517.

-3 Maclullich AM, Wardlaw JM, Ferguson KJ, Starr JM, Seckl JR, Deary IJ: Enlarged perivascular spaces are associated with cognitive function in healthy elderly man. J Neurol Neurosurg Psychiatry 2004;75:1519-1523.

$\checkmark 4$ Rouhl RP, van Oostenbrugge RJ, Knottnerus IL, Staals JE, Lodder J: Virchow-Robin spaces relate to cerebral small vessel disease severity. J Neurol 2008;255:692-696.

5 Hiroki M, Miyashita K: Linear hyperintensity objects on magnetic resonance imaging related to hypertension. Cerebrovasc Dis 2001;11:164-168.

6 Patankar TF, Mitra D, Varma A, Snowden J, Neary D, Jackson A: Dilatation of the Virchow-Robin space is a sensitive indicator of cerebral microvascular disease: study in elderly patients with dementia. Am J Neuroradiol 2005;26:1512-1520.

-7 Etemadifar M, Hekmatnia A, Tayari N, Kazemi M, Ghazavi A, Akbari M, Maghzi AH: Features of Virchow-Robin spaces in newly diagnosed multiple sclerosis patients. Eur J Radiol 2011;80:e104-e108.

8 Wuerfel J, Haertle M, Waiczies H, Tysiak E, Bechmann I, Wernecke KD, Zipp F, Paul F: Perivascular spaces - MRI marker of inflammatory activity in the brain? Brain $2008 ; 131$ : 2332-2340.

9 Achiron A, Faibel M: Sandlike appearance of Virchow-Robin spaces in early multiple sclerosis: a novel neuroradiologic marker. Am J Neuroradiol 2002;23:376-380.
0 Prineas J: Pathology of the early lesion in multiple sclerosis. Hum Pathol 1975;6:531554.

11 Braffman BH, Zimmerman RA, Trojanowski JQ, Gonatas NK, Hickey WF, Schlaepfer WW: Brain MR: pathologic correlation with gross and histopathology. 1. Lacunar infarction and Virchow-Robin spaces. AJR Am J Roentgenol 1988;151:551-558.

12 Heier LA, Bauer CJ, Schwartz L, Zimmerman RD, Morgello S, Deck MD: Large Virchow-Robin spaces: MR-clinical correlation. Am J Neuroradiol 1989;10:929-936.

13 Doubal FN, MacLullich AM, Ferguson KJ, Dennis MS, Wardlaw JM: Enlarged perivascular spaces on MRI are a feature of cerebral small vessel disease. Stroke 2010;41:450454.

14 Ransohoff RM, Kivisakk P, Kidd G: Three or more routes for leukocyte migration into the central nervous system. Nat Rev Immunol 2003;3:569-581.

15 Becker NH, Novikoff AB, Zimmerman HM: Fine structure observations of the uptake of intravenously injected peroxidase by the rat choroid plexus. J Histochem Cytochem 1967; 15:160-165.

16 Esiri MM, Gay D: Immunological and neuropathological significance of the VirchowRobin space. J Neurol Sci 1990;100:3-8.

17 Matsumoto Y, Fujiwara M: The immunopathology of adoptively transferred experimental allergic encephalomyelitis (EAE) in Lewis rats. I: Immunohistochemical examination of developing lesions of EAE. J Neurol Sci 1987;77:35-47.

18 Maclullich AM, Wardlaw JM, Ferguson KJ, Starr JM, Seckl JR, Deary IJ: Enlarged perivascular spaces are associated with cognitive function in healthy elderly men. J Neurol Neurosurg Psychiatry 2004;75:1519-1523.
19 Ferguson SC, Blane A, Perros P, McCrimmon RJ, Best JJ, Wardlaw J, Deary IJ, Frier BM: Cognitive ability and brain structure in type 1 diabetes: relation to microangiopathy and preceding severe hypoglycemia. Diabetes 2003;52:149-156.

20 Zhu YC, Tzourio C, Soumaré A, Mazoyer B, Dufouil C, Chabriat H: Severity of dilated Virchow-Robin spaces is associated with age, blood pressure, and MRI markers of small vessel disease: a population-based study. Stroke 2010;41:2483-2490.

21 Raine CS, Snyder DH, Valsamis MP, Stone $\mathrm{SH}$ : Chronic experimental allergic encephalomyelitis in inbred guinea pigs: an ultrastructural study. Lab Invest 1974;31:369380.

22 Greter M, Heppner FL, Lemos MP, Odermatt BM, Goebels N, Laufer T, et al: Dendritic cells permit immune invasion of the CNS in an animal model of multiple sclerosis. Nat Med 2005;11:328-334.

-23 McMahon EJ, Bailey SL, Castenada CV, Waldner H, Miller SD: Epitope spreading initiates in the CNS in two mouse models of multiple sclerosis. Nat Med 2005;11:335-339.

24 Becher B, Bechmann I, Greter M: Antigen presentation in autoimmunity and CNS inflammation: how $\mathrm{T}$ lymphocytes recognize the brain. J Mol Med 2006;84:532-543.

25 Bechmann I, Galea I, Perry VH: What is the blood-brain barrier (not)? Trends Immunol 2007;28:5-11.

26 Vos CM, Geurts JJ, Montagne L, van Haastert ES, Bö L, van der Valk P, Barkhof F, de Vries HE: Blood-brain barrier alterations in both focal and diffuse abnormalities on postmortem MRI in multiple sclerosis. Neurobiol Dis 2005;20:953-960. 